\title{
Transcranial and pulsed focused ultrasound that activates brain can accelerate remyelination in a mouse model of multiple sclerosis
}

\author{
T. A. Olmstead ${ }^{1}$, P. A. Chiarelli ${ }^{1}$, D. J. Griggs ${ }^{2}$, A. M. McClintic ${ }^{1}$, A. N. Myroniv ${ }^{1}$ and P. D. Mourad ${ }^{1,2^{*}}$
}

\begin{abstract}
Background: Multiple sclerosis (MS) impacts approximately 400,000 in the United States and is the leading cause of disability among young to middle aged people in the developed world. Characteristic of this disease, myelin within generally focal volumes of brain tissue wastes away under an autoimmune assault, either inexorably or through a cycle of demyelination and remyelination. This centrally located damage produces central and peripheral symptoms tied to the portion of brain within the MS lesion site. Interestingly, Gibson and colleagues noted that optical activation of transgenically tagged central neurons increased the thickness of the myelin sheath around those neurons. Since ultrasound, delivered transcranially, can also activate brain focally, we hypothesized that ultrasound stimulation that followed the temporal pattern of Gibson et al. applied to MS lesions in a mouse model might either decelerate the demyelination phase or accelerate its remyelination phase.

Methods: We created a temporal pattern of ultrasound delivery that conformed to that of Gibson et al. and capable of activating mouse brain. We then applied ultrasound, transcranially, following that temporal pattern to separate cohorts of a mouse model of multiple sclerosis, using three different ultrasound carrier frequencies $(0.625 \mathrm{MHz}, 1.09 \mathrm{MHz}, 2.0$ $\mathrm{MHz}$ ), during each of the demyelinating and remyelinating phases. After identifying the most promising protocol and MS brain state through qualitative analysis of myelin content, we performed additional studies for that condition then assayed for change in myelin content via quantitative analysis.
\end{abstract}

Results: We identified one ultrasound protocol that significantly accelerated remyelination, without damage, as demonstrated with histological analysis.

Conclusion: MRI-guided focused ultrasound systems exist that can, in principle, deliver the ultrasound protocol we successfully tested here. In addition, MRI, as the clinical gold standard, can readily identify MS lesions. Given the relatively low intensity values of our ultrasound protocol - close to FDA limits - we anticipate that future success with this approach to MS therapy as tested using more realistic MS mouse models may one day translate to clinical trials that help address this devastating disease.

Keywords: Focused ultrasound, Neuromodulation, Brain activation, Multiple sclerosis, Therapy

\footnotetext{
* Correspondence: doumitt@uw.edu

${ }^{1}$ Department of Neurological Surgery, University of Washington, Seattle, WA 98195, USA

2Division of Engineering and Mathematics, University of Washington, Bothell, WA 98011, USA
}

(c) The Author(s). 2018 Open Access This article is distributed under the terms of the Creative Commons Attribution 4.0 International License (http://creativecommons.org/licenses/by/4.0/), which permits unrestricted use, distribution, and reproduction in any medium, provided you give appropriate credit to the original author(s) and the source, provide a link to the Creative Commons license, and indicate if changes were made. The Creative Commons Public Domain Dedication waiver (http://creativecommons.org/publicdomain/zero/1.0/) applies to the data made available in this article, unless otherwise stated. 


\section{Introduction}

Multiple sclerosis (MS) represents the leading cause of disability among young to middle aged people in the developed world [1], with over 400,000 people in the United States affected by it [2]. Its characteristic loss of central and peripheral function arises due to an autoimmune assault on the myelin in the brain, often with an initial 'relapsing-remitting' phase (with attendant loss and at least partial recovery of function) followed by net degradation of myelin and function [2].

Interestingly, a range of studies show that the quality and quantity of myelin around a given axon depends upon the activation of that axon [3, 4]. Using a disease-free optogenetic mouse model, a more recent study demonstrated enhanced myelin buildup around axons activated by pulsed laser light delivered via fiber optic cable with a specific temporal pattern [5]. Finally, several studies show that transcranial delivery of pulsed focused ultrasound (pFU) can non-destructively activate central neural circuits across a range of species, including mice [6], rats [7], rabbits [8], sheep [9], non-human primates [10] and humans [11], reviewed recently in a comprehensive fashion [12].

Taken together, these citations motivated the hypothesis governing the present work: targeted transcranial pFU stimulation of axons within MS lesions in a manner that follows the laser-light temporal pattern of Gibson et al. (2014) - (reference [5]) - can decrease these lesion's rate of demyelination and/or increase their rate of remyelination.
To test this hypothesis, we applied three pFU protocols, all with a temporal pattern that conformed to that delivered by Gibson et al., each with a different carrier frequency, and all with relatively low ultrasound intensity values, to one side of the brains of separate sets of a mouse model of MS. One set experienced chemically induced demyelination; the other set experienced remyelination after cessation of chemically induced demyelination. We used histopathological measures of myelin to determine the effect of pFU neural activation on myelin content, with the contralateral hemisphere serving as a control. We also stained for neuronal structure, to assay for possible damage created by the ultrasound.

\section{Methods}

Animal model

All animal procedures were approved by the University of Washington Institutional Animal Care and Use Committee under protocol \#4084-08 and conformed to applicable national guidelines.

Adult male C57BL/6 J mice weighing approximately 25-30 g (The Jackson Laboratory, Bar Harbor, ME) were used for all procedures.

Mice were fed a $0.2 \%$ Cuprizone diet (Envigo, Madison WI) following two separate feeding protocols, described below. The Cuprizone mouse model of demyelination produces multiple sclerosis-like reduction in myelin primarily in the corpus callosum and superior cerebellar peduncles [13-16]. These effects are visible via $\mathrm{T}_{2}$-weighted $\mathrm{MRI}$ after 4 weeks of
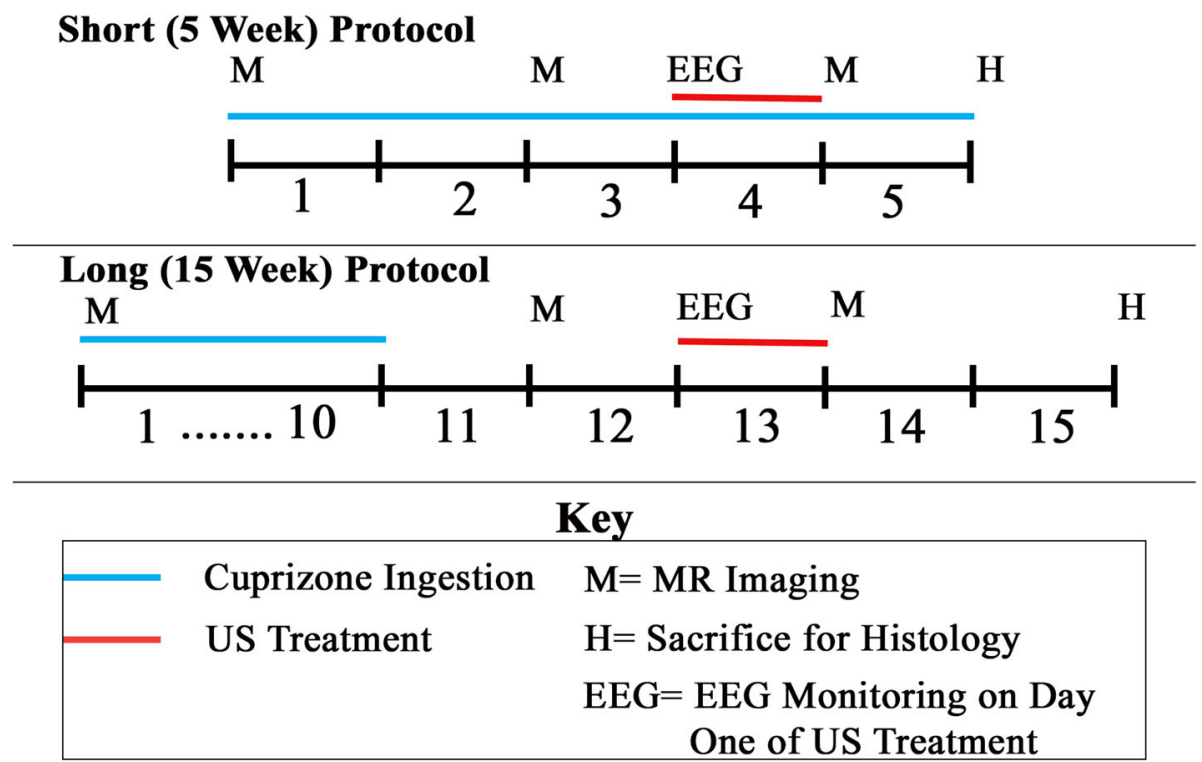

Fig. 1 Map of the time course of the experimental procedures. We used two experimental protocols: a 'short, 5 week' protocol that tested ultrasound's effect on demyelination and a 'long, 15 week' protocol that tested ultrasound's effect on remyelination. Both protocols required ultrasound application every day for a week, when appropriate. They differed by the timing of application of Cuprizone chow, ultrasound treatment, MRI imaging and EEG monitoring 
Cuprizone administration [14], with full demyelination occurring by 6 weeks [17]. Cuprizone-induced demyelination can switch to spontaneous remyelination as early as 4 days after Cuprizone withdrawal [16], making it convenient for studying therapies that may prevent demyelination as well as accelerate remyelination [18]. We used two separate protocols for Cuprizone

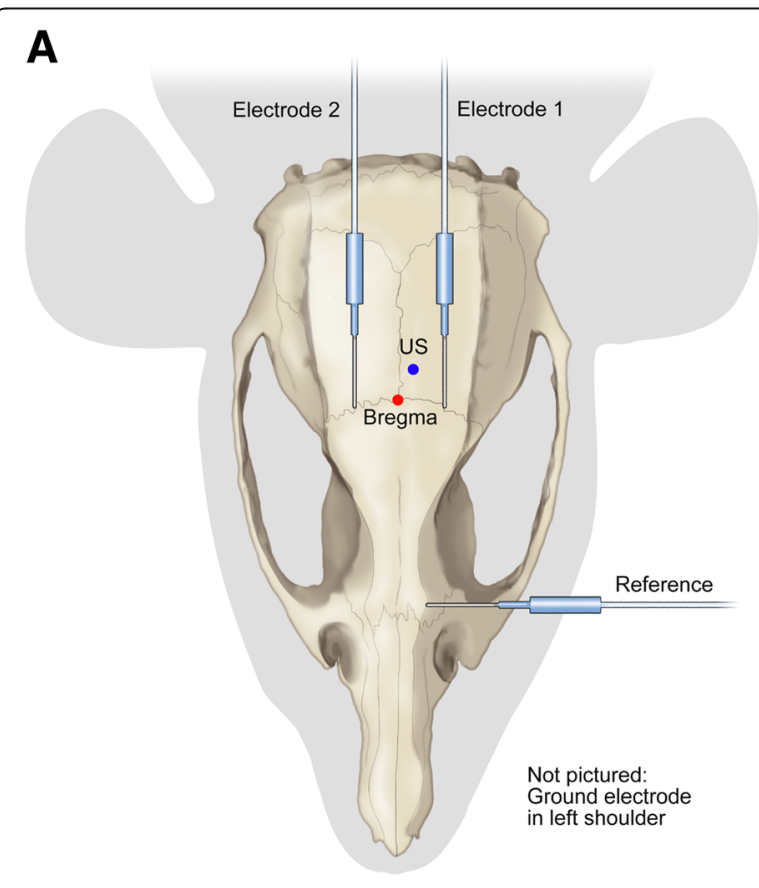

B

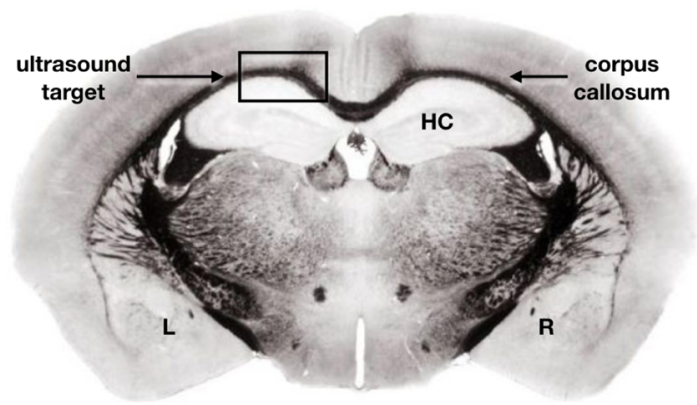

Fig. 2 Global view of the experiment. a Plan view. We delivered ultrasound transcutaneously and transcranially to the left side of mouse brain, using a micro-positioner to deliver that ultrasound to $-1.03 \mathrm{~mm}$ behind Bregma, $0.5 \mathrm{~mm}$ to the right of center, and $0.5 \mathrm{~mm}$ below the skull - within the corpus callosum, below the somatosensory cortex and above the hippocampus. We placed two subdermal electrodes on either side of the ultrasound focus. We placed a reference electrode rostral to the site of ultrasound application, and a grounding electrode subcutaneously on the mouse's shoulder. b Coronal view. This schematic shows a coronal view of mouse brain, stained for myelin, at $-1.03 \mathrm{~mm}$ Bregma, highlighting the corpus callosum (the 'eye brow-like' black and curved line), the hippocampus ( $\mathrm{HC}$ ) and the point of ultrasound exposure administration (Fig. 1). For the first, short, 5-week protocol, mice received Cuprizone chow for the entire 5 weeks, which generated demyelination. For the second, long, 15-week protocol, mice received Cuprizone chow for the first 10 weeks then reverted to standard chow, thereby allowing remyelination after 10 weeks. With the 5 -week protocol we sought to test the ability of pFU-activation of brain to slow demyelination. With the 15-week protocol we sought to test the ability of pFU-activation of brain to increase the rate of remyelination.

\section{Ultrasound application - General considerations}

Ultrasound was applied (with instruments, protocols and time points described below) while animals were under anesthesia as follows: we induced an initial deep anesthesia through a injection of a mixture of ketamine $(86.7 \mathrm{mg} / \mathrm{Kg})$ and xylazine $(9.3 \mathrm{mg} / \mathrm{Kg})$, with supplemental doses administered as needed to maintain an even anesthetic plane. Toe pinches were used to check depth of anesthesia. A circulating water heating pad (Gaymar Industries, Orchard Park, NY) set to $100^{\circ} \mathrm{F}$ maintained animal body temperature during the procedure. After induction of anesthesia, we placed the mice in a stereotaxic headpiece. To facilitate ultrasound transmission through the skull, hair was removed from the top of each mouse's head using shears and depilatory cream. Through use of a sub-millimeter resolution micro-positioner, we placed the ultrasound's focus in the left hemisphere of the mouse's brain such that it overlapped with the center of the corpus callosum, itself under the somatosensory cortex and above the hippocampus - Fig. 2.

\section{Ultrasound sources}

We used three different transducers for pFU-based brain activation, depending upon the specific study as described below, with each choice motivated by existing ultrasound systems, two of which are ready now for application to human brain. For the lowest ultrasound frequency, we used a custom-made focused ultrasound transducer with center frequency $0.625 \mathrm{MHz}$ and accompanying matching network (Applied Physics Laboratory, University of Washington, Seattle WA), consistent with the Insightec ExAblate MRI-guided ultrasound device currently available for treatment of brain $[19,20]$. Our mid-frequency system had a carrier frequency of $1.09 \mathrm{MHz}$ with an accompanying matching network (Model Number $\mathrm{H}-$ 101, Sonic Concepts, Woodinville, WA), close to the 1.0 $\mathrm{MHz}$ carrier frequency of the Sonalleve MRI-guided therapeutic ultrasound device, developed by Philips Ultrasound, now held by Profound Medical [21] and another, similar MRI-guided therapeutic device developed by 
Imasonic [22]. We also used a higher-frequency focused ultrasound transducer, with a carrier frequency of 2.0 $\mathrm{MHz}$ (Model Number $\mathrm{H}-148$ Annular Array, Sonic Concepts, Woodinville, WA), consistent with existing transcranial Doppler devices, hence capable of transcranial delivery though currently without MRI guidance. We used a calibrated needle hydrophone (HNR-1000, Onda Corporation, Sunnyvale, CA) placed in a tank filled with deionized, degassed water to calibrate all transducers. We drove our transducers using an appropriate arrangement [7] of three function generators (HP33120A, HewlettPackard, Palo Alto, CA, USA), an oscilloscope (Wave Runner LT 322 oscilloscope, LeCroy Corporation, Chestnut Ridge, NY, USA), and an amplifier (ENI Model A150 RF Power Amplifier, ENI, Rochester, NY).

\section{Ultrasound and Cuprizone protocol during demyelination (short, 5-week protocol)}

Cohorts of 3-4 mice received Cuprizone chow for a total of 5 weeks, without reverting to standard chow (again, Fig. 1). pFU was applied for five consecutive days during the fourth week of Cuprizone treatment before complete demyelination in an attempt to decelerate or even reverse demyelination. Following this therapeutic protocol, the mice recovered for 2 weeks before euthanasia to facilitate histology.

\section{Ultrasound and Cuprizone protocol during remyelination studies (long, 15-week protocol)}

Cohorts of 3-4 mice received Cuprizone chow for 10 weeks (Fig. 1). After 10 weeks, animals were then fed standard chow, subsequently experiencing remyelination. After an additional 2 weeks, hence during week 13, pFU was applied to the mice each day for 5 days in order to test the ability of pFU to accelerate remyelination. Following this therapeutic protocol, the mice recovered for 2 weeks before euthanasia to facilitate histology.

\section{Ultrasound pulse sequence and intensity for 5- and 15- week protocols}

Figure 3 shows the ultrasound application pattern, whose temporal envelope mimics that of Gibson et al. (2014). Specifically, that envelope consists of 20 stimulations per second lasting for $30 \mathrm{~s}$, followed by a rest period of $90 \mathrm{~s}$, repeated for a total of $30 \mathrm{~min}$ per day, for each of 5 days.

Note that Gibson et al. applied continual light pulses, each lasting 25 milliseconds ( $\mathrm{ms}$ ) and followed by no light for $25 \mathrm{~ms}, 20$ times per second. While continually applied ultrasound can activate brain [23], we followed the majority of papers (reviewed recently in Bobola et al. [12]) as well as our own work [7, 24], by stimulating brain with a set of rapidly applied ultrasound bursts for each of the 20 times per second within the Gibson et al.

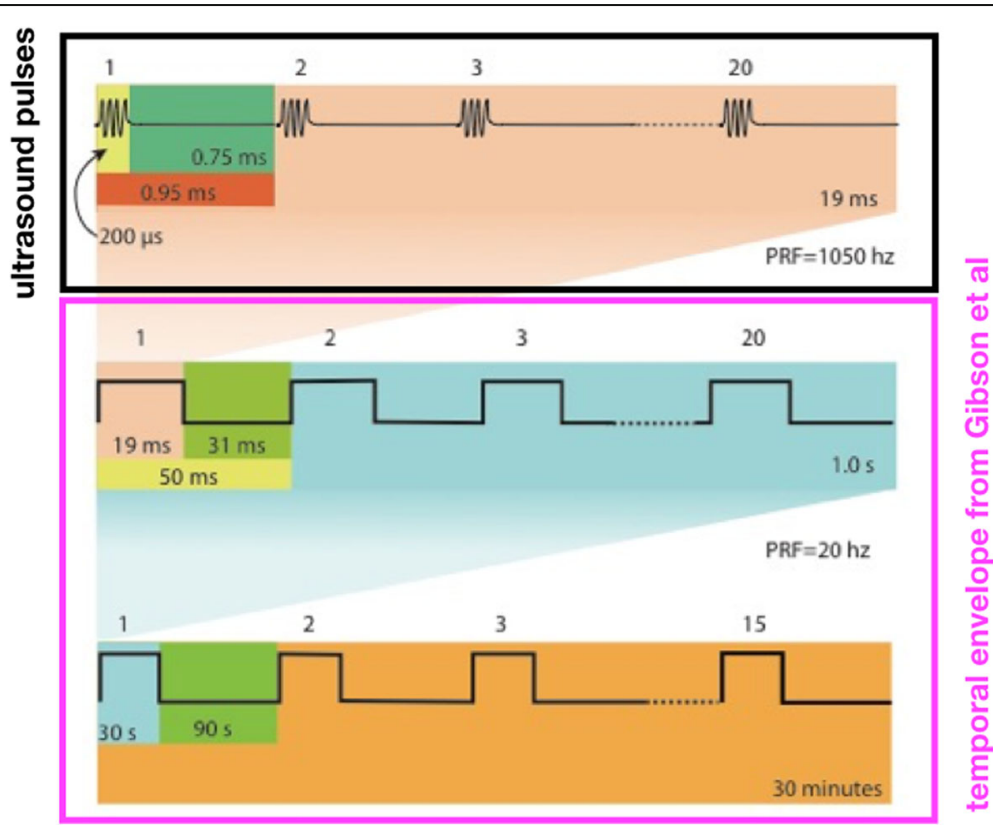

Fig. 3 Temporal pattern of ultrasound delivery, modeled after the optical stimulation pattern of Gibson et al. The optical protocol delivered light twenty times per second, with each pulse of light lasting 25 milliseconds (ms), for a total of $30 \mathrm{~s}$. Each 30-s long period of pulsed stimulation preceded a 90-s long period of no stimulation. These on-off periods of light exposure lasted a total of 30 min per day, applied for five contiguous days. We modified this protocol to application of ultrasound by using pulsed ultrasound during each of 19-ms long time periods, followed by 31 ms of no ultrasound, rather than continual light for 25 ms followed by 25 ms of no light. The figure here shows 20 ultrasound pulses applied during each $19 \mathrm{~ms}$ time period, each pulse lasting 200 microseconds 
protocol. Specifically, 20 times per second, we applied a set of 20 very short pulses of ultrasound (each lasting 200 microseconds) at a pulse repetition frequency (PRF) of $1050 \mathrm{~Hz}$. This ultrasound stimulation burst lasted for $19 \mathrm{~ms}$ followed by $31 \mathrm{~ms}$ of no light (Fig. 3, top row). Each ultrasound stimulation burst was then repeated at $20 \mathrm{~Hz}$ for $30 \mathrm{~s}$ (30 s of pulsed ultrasound "on"), with a rest-period of $90 \mathrm{~s}$ of no ultrasound ( $90 \mathrm{~s}$ of ultrasound "off") - Fig. 3, middle and bottom row. This continued for $30 \mathrm{~min}$ per ultrasound session, with one session per day over a five-day period. This ultrasound scheme was used for each of the 5- and 15-week experimental protocols.

With regard to intensity, we performed exploratory studies with naive mice to identify a single ultrasound intensity value that produced reliable brain activation across all three ultrasound carrier frequencies. That analysis yielded ultrasound with a spatial peak intensity averaged over each 200 microsecond long pulse of $1.52 \mathrm{~W} / \mathrm{cm}^{2}\left(\mathrm{I}_{\text {sppa }}\right)$. Twenty of them applied over 19 milliseconds (a single ultrasound burst) had a spatial peak burst average intensity of $3.2 \mathrm{~W} / \mathrm{cm}^{2}$ $\left(\mathrm{I}_{\text {spba }}\right)$ or, averaged over time instead of over a burst, $0.06 \mathrm{~W} / \mathrm{cm}^{2}$, spatial peak temporal average intensity $\left(\mathrm{I}_{\text {spta }}\right)$. With 20 such ultrasound stimulation bursts per second, we therefore used $1.2 \mathrm{~W} / \mathrm{cm}^{2}\left(\mathrm{I}_{\text {spta }}\right)$ within the complex temporal pattern shown in Fig. 3, not far above the FDA guideline for diagnostic ultrasound
[25] of $0.72 \mathrm{~W} / \mathrm{cm}^{2}\left(\mathrm{I}_{\mathrm{spta}}\right)$ and consistent with intensity values applied to anesthetized rats that changed their response to anesthesia [26].

\section{EEG monitoring}

We monitored brain activity using subdermal electrodes (with details below) on only the first of 5 days of ultrasound application. In this way we minimized discomfort for the animal and avoiding implanted electrodes, which would have interfered with the MRI imaging. During a given EEG session, time-series recordings were first obtained for $4 \mathrm{~min}$, without any ultrasound - the baseline recording. Next came application of ultrasound for $30 \mathrm{~min}$, with the specific temporal pattern as described in Fig. 3. For each 30-min EEG recording corresponding to a single ultrasound protocol, the time-series was dissected into 50 millisecond (ms) windows during which time ultrasound was on during the first $19 \mathrm{~ms}$, and off during the remaining $31 \mathrm{~ms}$. We designate that second, off period as 'background'.

We used two subdermal EEG electrodes, arranged in a single-channel bipolar configuration, with a third electrode for reference and a fourth for ground. Figure $2 \mathrm{a}$ shows the locations of the subdermal needle electrodes and their relation to landmarks on a mouse skull. Our EEG setup used thin wire EEG electrodes (Ambu Neuroline Subdermal 27G, Cadwell, Kennewick, WA) recorded at a sampling rate of $38,400 \mathrm{~Hz}$ on a 16 -channel biosignal

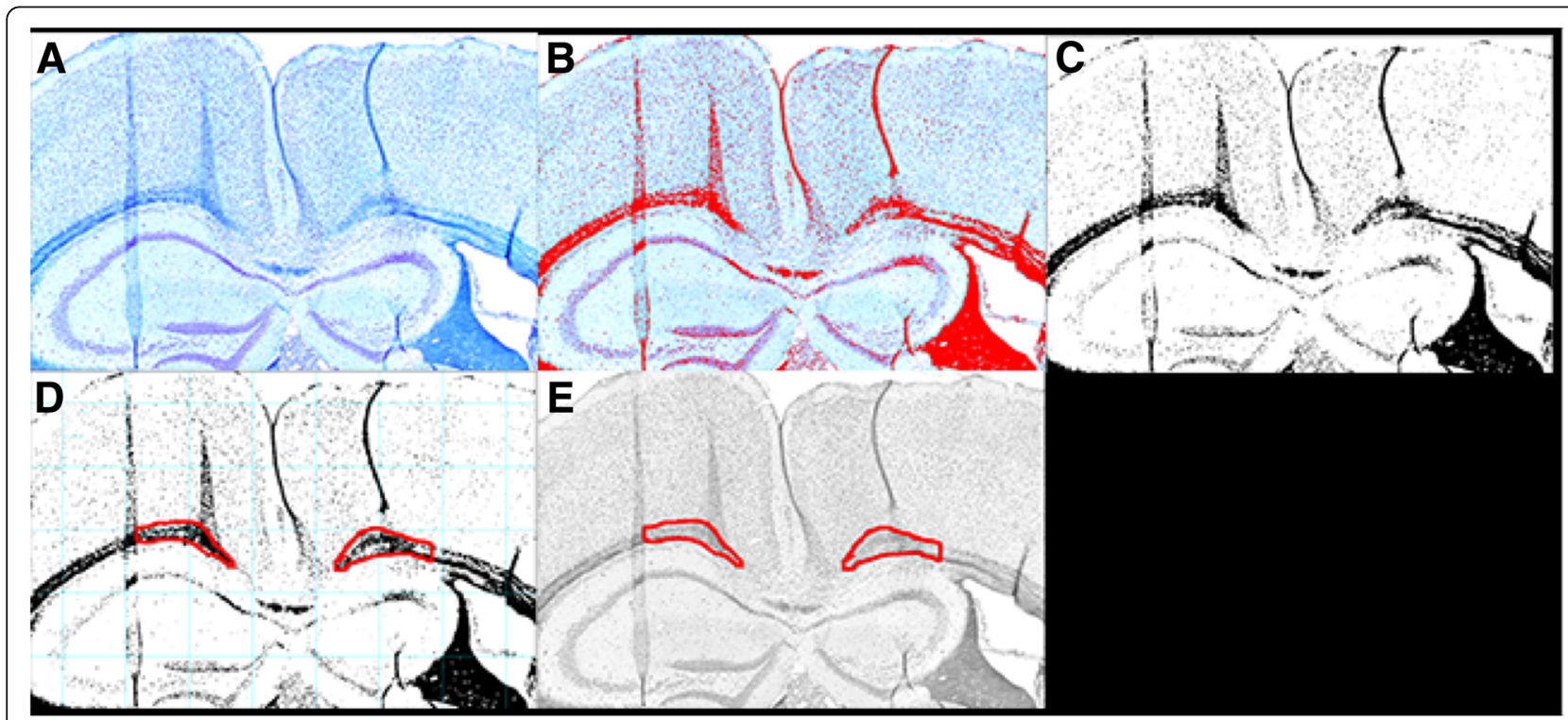

Fig. 4 Analysis method of histology images of myelin. a Original color brightfield image. Note that the left portion of these brain images correspond to the left hemisphere of the mouse brain. $\mathbf{b}$ Color thresholding (shown in red) on the same image. Thresholding was done in color since saturation for myelinated tissues showed variation relative to unmyelinated regions. c Resulting black and white mask derived from (b). d Black and white mask with grid and both regions of interest outlined in red. Note that the left outline does not extend past the second tissue fold (anomaly), and that the length of the right outline matches that of the left, by design. e 8-bit black and white original image with both ROls overlaid in red. We quantify the grey values indicative of myelin from this image, with darker hence more grey pixels associated with more myelin 
amplifier (gUSBamp, Guger Technologies OG, Graz Austria) that integrated with Simulink (MathWorks, Natick MA). EEG monitoring and analysis was performed during ultrasound application. EEG results were analyzed using MATLAB software (MathWorks, Natick MA) and reported as an average plus standard error over each 19 millisecond pulses over the entire time of ultrasound application, a total of $\{20$ bursts/s $\}$ $\mathrm{X}\{30 \mathrm{~s} /[2 \mathrm{~min}]\} \quad \mathrm{X}\{30 \mathrm{~min} /$ day $\} \quad \mathrm{X}\{5$ days of ultrasound application $\}=45,000$ bursts.

\section{Magnetic resonance imaging (MRI)}

$\mathrm{T}_{2^{-}}$weighted baseline MRI scans were obtained before administering Cuprizone on the first of the first week of both the 5- and 15-week MS protocols. Scans were also collected during the week before pFU treatment (pre-US), and the week after pFU treatment (post-US), as represented in Fig. 1. All scans were conducted at the University of Washington High Resolution Imaging (HRIM) facility using a 14 Tesla $(600 \mathrm{MHz})$ vertical wide bore Bruker Magnetic Resonance Spectrometer (Bruker Corporation, Billerica MA) with integral temperature control and isoflurane anesthesia capability. Resultant DICOM image files were converted to standard image formats (tiff) using Adobe Photoshop CC 2017 (Adobe Systems Incorporated, San Jose CA). T2 parameters follow that of Thiessen et al. (2013) (reference [14]).

\section{Histology and image analysis}

At the completion of the study, all 5- and 15-week MS protocol animals were transcardially perfused in $10 \%$ Neutral Buffered Formalin (Fisher Protocol, Fisher Scientific, Hampton NH) after induction of deep anesthesia with isoflurane. Following perfusion, animals were decapitated, and heads were post-fixed in Formalin for at least 1 week before brain removal for sectioning and staining.

\section{Histological staining}

After sufficient fixation, the brains were sectioned at $12 \mu \mathrm{m}$, and mounted onto Superfrost Plus microscope slides (Fisherbrand, Fisher Scientific, Hampton NH). These slides were then stained, one stain per slide, with Luxol fast blue for myelin, or Cresyl Violet (a neuronal stain) to assay for cellular damage.

\section{Histological image acquisition}

Histological images were taken using a Nikon Eclipse TE200 inverted microscope with a Nikon DS-Fi1 camera, a Nikon 0.6x TV lens, and a 4x magnification objective. The images were collected in color using unfiltered bright-field settings. Due to the method in which sections were collected and mounted, the left side of each slide corresponds to the left side of the resultant image, and also corresponds to the left side of the animal itself, where we applied ultrasound.

\section{Histological image analysis}

All analysis was done using the Fiji version of ImageJ [27] - Fig. 4. Myelin-stained images were loaded into ImageJ, then thresholded in color, and scaled globally to achieve 0.32 pixels $/ \mu \mathrm{m}$. Next, a black and white mask of the image was created from the thresholded image. Myelinated areas within the corpus callosum region of interest were then outlined as follows. These outlines were done as conservatively as possible using the lasso tool: if an anomaly occurred on one side, outlines were extended to but not exceeding the anomaly, and the length of the outline on the contralateral side was measured to not exceed the maximum length of the constrained side. After outlining, the original image was converted to 8-bit greyscale, and both outlined ROIs were overlaid on top. Using the ROI manager in ImageJ, the measure functionality was used to determine the area, mean grey value, and maximum

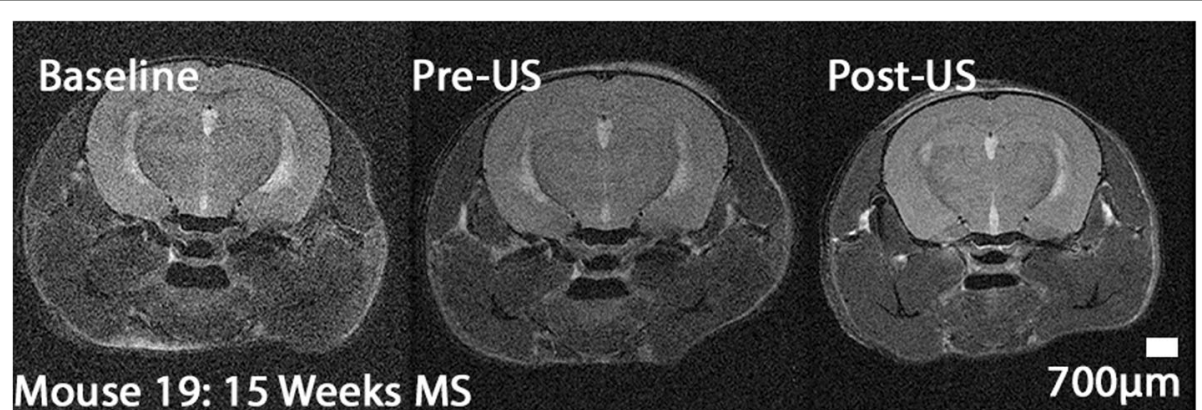

Fig. $5 \mathrm{MRI}$ imaging of long protocol (15 week) mice. This image demonstrates the presence of MS-like pathology, specifically decreased myelin in the corpus callosum, relative to their baseline images taken before introduction of Cuprizone, for a representative mouse brain exposed to 1.09 $\mathrm{MHz}$. Note that the left portion of a given MRI image corresponds to the left hemisphere of that mouse brain 


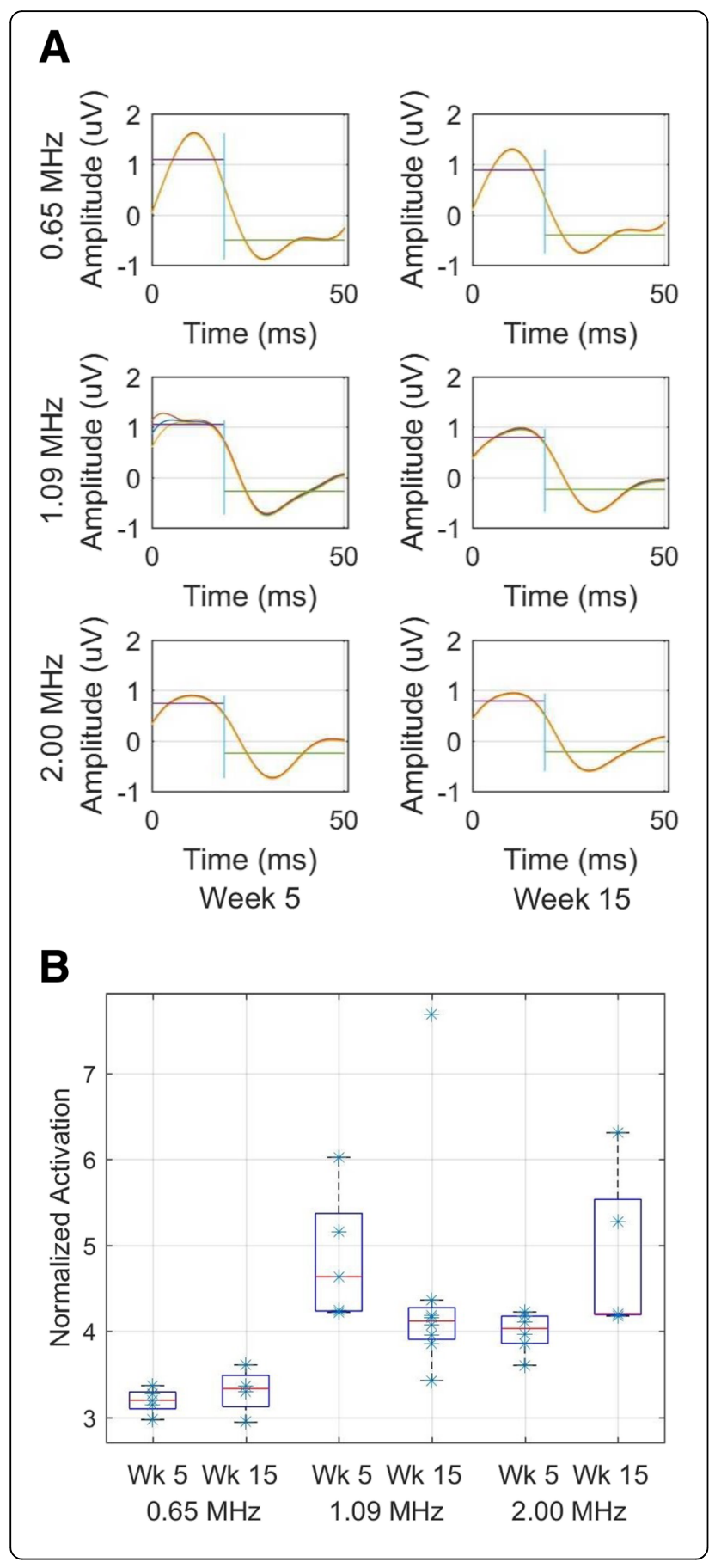

Fig. 6 Ultrasound applied to MS-model mice successfully activates brain. a Mean and standard error of EEG signals recorded from a representative mouse within each MS and ultrasound protocol. Horizontal lines denote either the mean value of the EEG signal during ultrasound application, hence observed brain activation (the first $19 \mathrm{~ms}$ ) or the mean value of the EEG signal during the time without ultrasound (the 'background', during the subsequent $31 \mathrm{~ms}$ ). b Calculated for every 50-ms pair in the time series for each mouse, and aggregated across all mice for each protocol, this figure's boxplots show the difference between mean brain activity induced by ultrasound and mean background brain activity, normalized by the absolute value of the mean background brain activity. $(0.625 \mathrm{MHz}, n=4 ; 1.09 \mathrm{MHz}$, $n=4 ; 2.0 \mathrm{MHz}, n=4$ )

and minimum grey values from the greyscale image with the ROI overlays.

Pixel darkness was compared (left [ultrasound] versus right [control]) within a single image using the original, unmodified mean grey values from the ROI overlays in the Image J analysis. From this, a percent difference in myelin content was derived by calculating the difference between the average gray value for the left side minus that of the right side, divided by gray value from the right (control) side.

\section{Pilot then final study with MS mice}

We performed a screening study with separate cohorts of demyelinating and remyelinating MS mice to facilitate qualitative histopathological analysis of the myelin content in the brain tissue samples associated with each ultrasound protocol (each one, again defined by the following carrier frequencies: 0.625 $\mathrm{MHz}, 1.09 \mathrm{MHz}$ and $2.0 \mathrm{MHz}$ ) - six sets of mice, each with $n=4$ mice/cohort. Through this qualitative analysis we identified the ultrasound protocol that had the greatest effect on myelin content for either or both of demyelinating and remyelinating states, then performed additional studies for that protocol on another three MS mice. We then pooled the histological results for that most promising ultrasound protocol applied to that demyelinating or remyelinating state, and performed the quantitative histological analysis described above on those seven mice.

\section{Results}

\section{MRI scans}

Short, 5 week protocol animals did not show significant change between baseline and pre-US MRI scans, consistent with model behavior and our decision to apply ultrasound before completion of demyelination (images not shown). Long, 15 week protocol animals showed significant demyelination during the remyelination phase in MRI images (Fig. 5) without, however, measurable changes in myelin content after ultrasound application (analysis not shown). 


\section{EEG analysis}

Both demyelinating (short, 5 week) and remyelinating (long, 15 week) studies demonstrated successful production of brain activation by ultrasound (Fig. 6a). In all cases, the mean amplitude recorded by EEG during ultrasound application was higher than that of the background. The two higher frequency protocols generated statistically significantly more brain activity than the low-frequency protocol for each of the 5-week and 15-week animals (Fig. 6b).

\section{Histological analysis}

Visual inspection of histological slides stained for myelin for all $(n=4)$ short protocol therapeutic animals indicated no difference between pFU therapy and control sides of the brain (images not shown). Visual inspection of all $(n=4)$ long, 15-week MS mice told a different story - see Fig. 7. Note that these representative results for $0.625 \mathrm{MHz}$, and $2.0 \mathrm{MHz}$ ultrasound protocol animals show no qualitative difference in myelin content between treated and untreated hemispheres, consistent with all the brain-tissue samples. However, visual inspection of our initial cohort of remyelinating MS mice exposed to $1.09 \mathrm{MHz}$ ultrasound suggested that these animals had more myelin within the hemisphere of brain where ultrasound was applied relative to the contralateral side.

Motivated by this promising qualitative result, we performed additional 15-week MS mouse studies $(n=3)$ using $1.09 \mathrm{MHz}$ ultrasound, aggregated those new histological data with our initial findings, and then performed a quantitative analysis of the histology. In addition to more myelin (e.g., Figs. 7b and 8a and a'), we did not observe any neuronal damage from ultrasound application in any of the 7 animals at $1.09 \mathrm{MHz}$ (e.g., Fig. $8 \mathrm{~b}$ and b').

Analyzing over all remyelinating mice exposed to 1.09 $\mathrm{MHz}$, Fig. 9 and Table 1 together show the result of quantitative analysis of the amount of myelin associated with this ultrasound protocol, demonstrating that the side of brain exposed to ultrasound had more myelin than the control side, with statistical significance.

\section{Discussion}

Gibson et al. observed that an optogenetic stimulatory paradigm could cause myelin to thicken around central neurons made amenable to light-based stimulation thanks to transgenic techniques. Others have observed activation of brain function created by transcranially delivered ultrasound. Taken together, these results motivated the present study, which sought to ascertain whether or not ultrasound could activate demyelinated and/or remyelinating brain and thereby enhance the amount of myelin in those brains. To do so, we translated the temporal pattern of the optically-based neuron stimulation protocol of Gibson et al. into an ultrasound-based brain activation protocol. Through this exploratory work we identified one ultrasound protocol that accelerated remyelination, as demonstrated with histological analysis. In addition, this protocol did not damage mouse brain, as also shown with histological analysis.

Why did we observe accelerated remyelination in the hemisphere of brain that received neuro-stimulatory ultrasound, but did not observe decelerated demyelination? Perhaps ultrasound's positive effect on myelin production can only have a net impact without the presence of the driving force for demyelination, here the cuprizone chow. Further work, with mouse models of MS based upon experimentally produced autoimmune disorder, itself treated or not treated during the study, represents a natural next step to explore this question as well as move this new therapeutic paradigm forward towards possible clinical tests.

\section{Limitations}

As just discussed, we used a food-based model of multiple sclerosis, rather than one based upon lesion development created by an errant autoimmune system [28], the latter consistent with the human condition [29]. Future work will make use of such a model, with

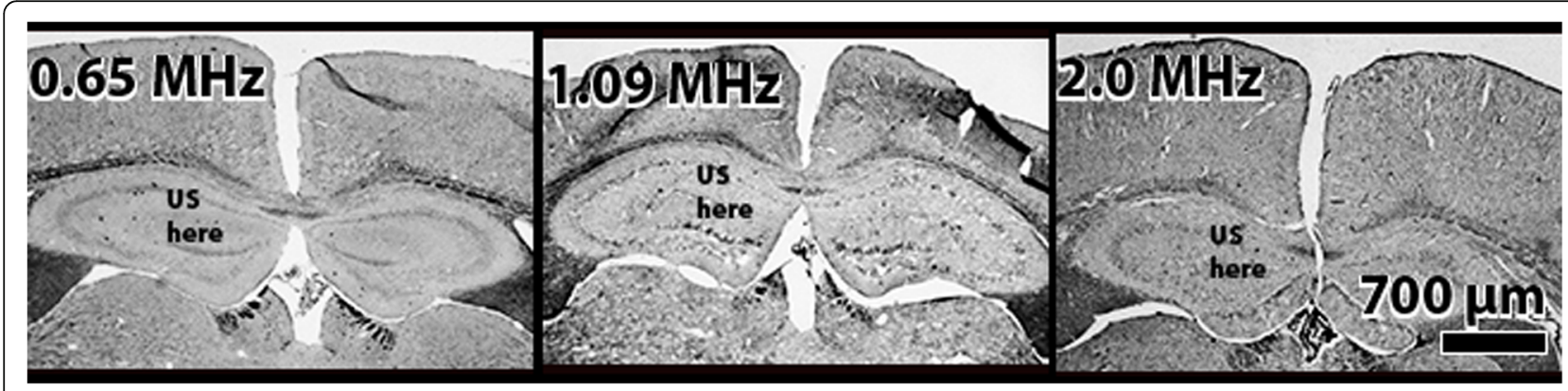

Fig. 7 Representative coronal slices of MS mouse brain stained for myelin for each ultrasound protocol, designated by the frequency of ultrasound. Note that the corpus callosum for only the $1.09 \mathrm{MHz}$ sample shows, qualitatively, enhanced myelin at the region of ultrasound application (above the annotation 'US here'). Note that the left portion of a given brain image corresponds to the left hemisphere of that mouse brain 


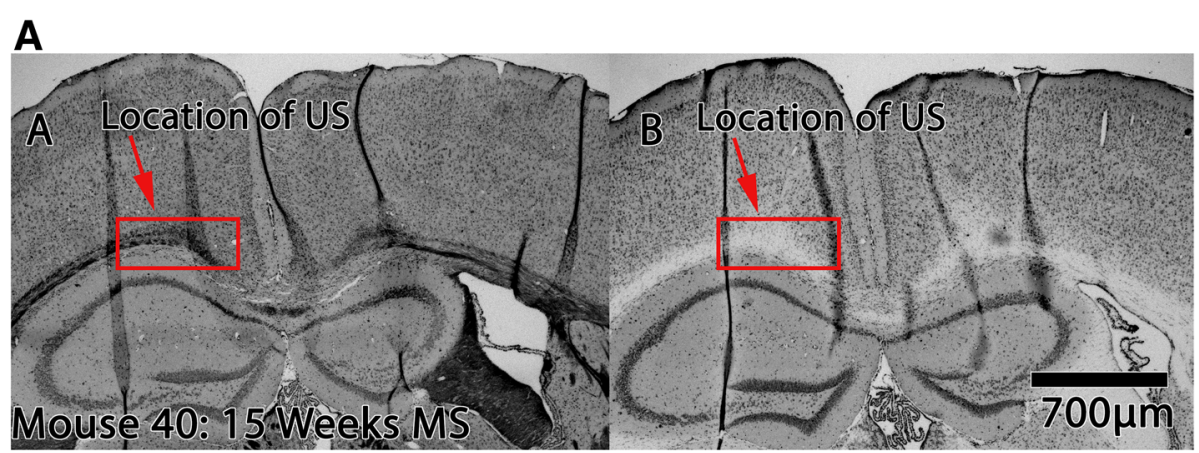

B

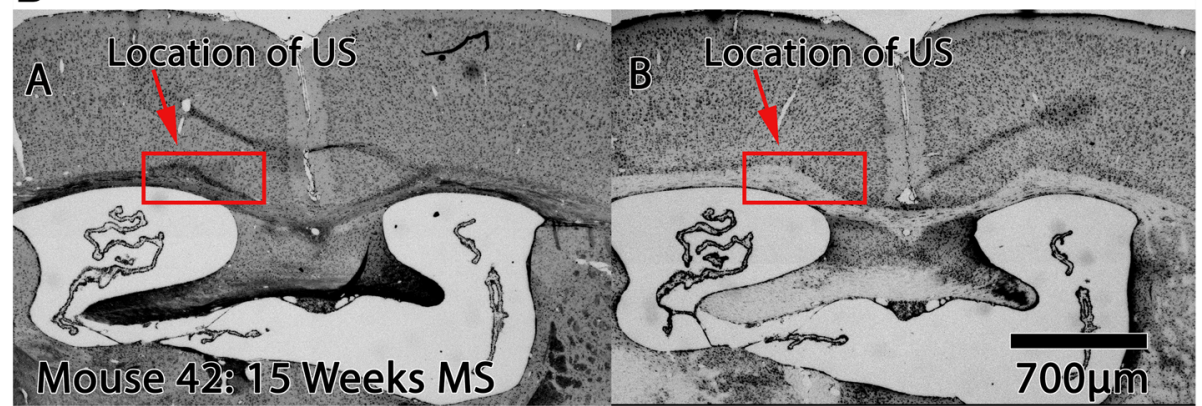

Fig. 8 Myelin and Cresyl violet stains of representative mouse brains. (A,A') Myelin stain of brain from representative $1.09 \mathrm{MHz}$ long-protocol animals during remyelination studies. $\left(B, B^{\prime}\right)$ Cresyl violet stain of adjacent slices of brain from the same animals as in $\left(A, A^{\prime}\right)$. Note that the left portion of a given brain image corresponds to the left hemisphere of that mouse brain

and without adjunctive therapy to fight the demyelination. Also, we used the portion of brain contralateral to ultrasound application as the control, leaving open the possibility that we may have activated the entire brain, hence increased myelin content on each side of the corpus collosum, though more efficiently ipsilateral to ultrasound application. Future studies with the autoimmune model will include a broader class of controls. Finally, we did not monitor for potential changes in behavior and function, the ultimate arbiters for success [30]. We intend to do so in the future.

Human skulls attenuate ultrasound much more than mouse skulls, and vary in structure both within a given human and between them. If, after further study, the $1.09 \mathrm{MHz}$ ultrasound continues to provide optimal therapeutic benefit, ultrasound delivery systems will have to compensate for thicker, and variable-thickness human skulls, a need met by existing systems [21, 22]. We have

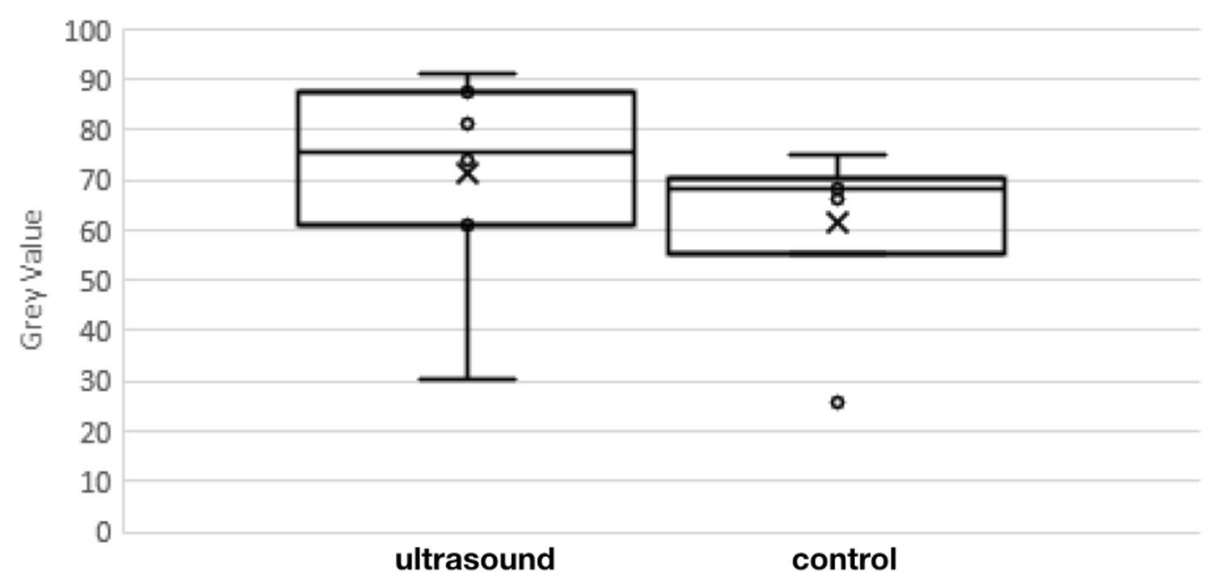

Fig. 9 Gray-scale value that measures extent of myelin on the left side of mouse brain to which ultrasound was applied compared to that of the right (control) side. Note higher values denote more myelin. This difference in myelin between brain hemispheres had statistical significance $(n=7$; two-sided, paired Student's t-test, $p<0.02$ ) with a substantial effect size (t score $=3.02$ ) 
Table 1 Histological Analysis. Pixel darkness value for ipsilateral (ultrasound) vs contralateral (control) sides, with a higher value marking more myelin, with maximum value equaling 255

\begin{tabular}{|c|c|c|c|c|c|}
\hline Animal Number & ipsilateral & contralateral & difference & $\%$ difference $^{\mathrm{a}}$ & interpretation \\
\hline \multicolumn{6}{|l|}{ Group 1} \\
\hline 1 & 30.6 & 25.7 & 4.9 & $19 \%$ & More myelin due to ultrasound \\
\hline 2 & 87.4 & 66.2 & 21.2 & $32 \%$ & More myelin due to ultrasound \\
\hline 3 & 90.9 & 68.5 & 22.4 & $33 \%$ & More myelin due to ultrasound \\
\hline 4 & 75.3 & 69.7 & 5.6 & $8 \%$ & More myelin due to ultrasound \\
\hline \multicolumn{6}{|l|}{ Group 2} \\
\hline 5 & 61.2 & 55.2 & 6 & $11 \%$ & More myelin due to ultrasound \\
\hline 6 & 74 & 75 & -1 & $-1 \%$ & More myelin away from ultrasound \\
\hline 7 & 81.3 & 70.2 & 11.1 & $16 \%$ & More myelin due to ultrasound \\
\hline Mean (All Animals) & $71.5+/-7.75$ & $61.5+/-6.39$ & $10+/-3.3$ & $16.6+/-4.7 \%$ & Net more myelin due to ultrasound \\
\hline
\end{tabular}

a "\% difference" $=$ difference between ipsilateral and contralateral myelin values divided by contralateral value then expressed as a percent

speculated above that ultrasound alone may not suffice to remyelinate brain due to the on-going autoimmune attack central to multiple sclerosis, that it's successful use may therefore require adjunctive anti-autoimmune treatment. Together, ultrasound and that adjunctive treatment may thus require chronic application, an expensive prospect given currently the high cost of MRI systems and their use. Future studies with an autoimmune mouse model must therefore involve study of chronic application of ultrasound and medication.

\section{Conclusion}

Multiple sclerosis can severely impact patients, without, unfortunately, sufficient means of treating this disease. We have demonstrated in a mouse model of MS that focused ultrasound, known to activate brain, accelerated remyelination of MS lesions. MRI can readily identify MS lesions and MRI-guided focused ultrasound systems exist that can, in principle, deliver the ultrasound protocol we successfully tested here. Given the existence of these devices and the relatively low intensity value of our efficacious ultrasound protocol $-1.2 \mathrm{~W} / \mathrm{cm}^{2}$, close to FDA limits for diagnostic ultrasound - we anticipate that future success with this approach to MS therapy using more realistic MS mouse models may 1 day translate rapidly to clinical trials that help address this devastating disease.

\section{Abbreviations}

EEG: electroencephalography; FDA: food and drug administration; $I_{\text {spba: }}$ spatial peak burst average intensity values; $I_{\text {sppa: }}$ spatial peak pulse average intensity

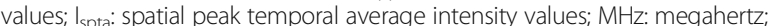
MRI: magnetic resonance imaging; ms: milliseconds; MS: multiple sclerosis; pFU: pulsed focused ultrasound; PRF: pulse repetition frequency

\section{Acknowledgements}

We thank Bobola MS, PhD, of the University of Washington's Department of Neurological Surgery for this statistical analysis; we also thank Kate Sweeny, who created for us the beautiful schematics that grace this paper. Finally, to create Fig. 2b we started with a figure from the High Resolution Mouse Brain Atlas (http://www.hms.harvard.edu/research/brain/atlas.html).

\section{Funding}

We are grateful for financial support from the Focused Ultrasound

Foundation, the primary funding agency for this work, as well as from $\mathrm{NIH}$ (1R21EY027557-01).

\section{Availability of data and materials}

The datasets used and/or analyzed during the current study are available from the corresponding author upon reasonable request.

\section{Authors' contributions}

Conceptualization and overall design (PDM); detailed experimental design (TAO, AMM, PDM); data acquisition (TAO, PAC, AMM, ANM); data analysis (TAO, PAC, DG, AMN, ANM); writeup (TAO, PDM) with input from all authors. All authors have reviewed and approve the contents of this paper.

Ethics approval and consent to participate

All animal studies were approved by the University of Washington's IACUC committee, protocol number 4084-08.

\section{Consent for publication}

Not applicable.

Competing interests

The authors declare that they have no competing interests.

\section{Publisher's Note}

Springer Nature remains neutral with regard to jurisdictional claims in published maps and institutional affiliations.

Received: 3 August 2018 Accepted: 15 November 2018

Published online: 10 December 2018

\section{References}

1. Koch-Henriksen N, Sørensen PS. The changing demographic pattern of multiple sclerosis epidemiology. Lancet Neurol. 2010;9(5):520-32.

2. Tullman MJ. Overview of the epidemiology, diagnosis, and disease progression associated with multiple sclerosis. Am J Manag Care [Internet]. 2013 Feb [cited 2018 Jul 26];19(2 Suppl):S15-20. Available from: http://www.ncbi.n/m.nih.gov/pubmed/23544716.

3. Ishibashi T, Dakin KA, Stevens B, Lee PR, Kozlov S V, Stewart CL, et al. Astrocytes promote myelination in response to electrical impulses. Neuron [Internet]. 2006 Mar 16 [cited 2018 Jul 27];49(6):823-32. Available from: http://linkinghub.elsevier.com/retrieve/pii/S0896627306000961

4. Jensen SK, Yong WW. Activity-Dependent and Experience-Driven Myelination Provide New Directions for the Management of Multiple Sclerosis. Trends Neurosci [Internet]. 2016 Jun [cited 2018 Jul 28];39(6):356-65. Available from: https://linkinghub.elsevier.com/retrieve/pii/S0166223616300030 
5. Gibson EM, Purger D, Mount CW, Goldstein AK, Lin GL, Wood LS, et al. Neuronal Activity Promotes Oligodendrogenesis and Adaptive Myelination in the Mammalian Brain. Science (80- ) [Internet]. 2014;344(6183). Available from: http://science.sciencemag.org/content/344/6183/1252304

6. Moore ME, Loft JM, Clegern WC, Wisor JP. Manipulating neuronal activity in the mouse brain with ultrasound : a comparison with optogenetic activation of the cerebral cortex. Neurosci Lett [Internet]. 2015;604:183-7 Available from: http://linkinghub.elsevier.com/retrieve/pii/S0304394015300446.

7. Mehić E, Xu JM, Caler CJ, Coulson NK, Moritz CT, Mourad PD. Increased anatomical specificity of neuromodulation via modulated focused ultrasound. PLoS One. 2014;9(2):e86939. https://doi.org/10.1371/journal. pone.0086939. eCollection 2014.

8. Yoo S-SS, Bystritsky A, Lee J-HH, Zhang Y, Fischer K, Min B-KK, et al. Focused ultrasound modulates region-specific brain activity. Neuroimage [Internet]. 2011 Jun 1 [cited 2018 Mar 11];56(3):1267-75. Available from: http://linkinghub.elsevier.com/retrieve/pii/ S105381191100214X

9. Lee W, Lee SD, Park MY, Foley L, Purcell-Estabrook E, Kim H, et al. Image-Guided Focused Ultrasound-Mediated Regional Brain Stimulation in Sheep. Ultrasound Med Biol [Internet]. 2016 Feb [cited 2018 Mar 19]; 42(2):459-70. Available from: http://linkinghub.elsevier.com/retrieve/pii/ S0301562915005876

10. Wattiez N, Constans C, Deffieux T, Daye PM, Tanter M, Aubry J-F, et al. Transcranial ultrasonic stimulation modulates single-neuron discharge in macaques performing an antisaccade task. Brain Stimul [Internet]. 2017 Nov [cited 2018 Jan 31];10(6):1024-31. Available from: http://www.ncbi.nlm.nih. gov/pubmed/28789857.

11. Legon W, Sato T, Opitz A, Mueller J, Barbour A, Williams A, et al. Transcranial focused ultrasound modulates the activity of primary somatosensory cortex in humans. Nat Neurosci [Internet]. 2014;17(2): 322-9 Available from: https://doi.org/10.1038/nn.3620.

12. Bobola MS, Chen L, Ezeokeke CK, Kuznetsova K, Lahti AC, Lou W, et al. A Review of Recent Advances in Ultrasound, Placed in the Context of Pain Diagnosis and Treatment. Curr Pain Headache Rep [Internet]. 2018 Sep 10 [cited 2018 Jul 27];22(9):60. Available from: http://link. springer.com/10.1007/s11916-018-0711-7

13. Goldberg J, Clarner T, Beyer C, Kipp M. Anatomical distribution of Cuprizone-induced lesions in C57BL6 mice. J Mol Neurosci [Internet]. 2015; 57(2):166-75 Available from: https://doi.org/10.1007/s12031-015-0595-5.

14. Thiessen JD, Zhang $Y$, Zhang $H$, Wang L, Buist R, Del Bigio MR, et al. Quantitative MRI and ultrastructural examination of the cuprizone mouse model of demyelination. NMR Biomed. 2013;26(11):1562-81.

15. Blakemore WF. Demyelination of the superior cerebellar peduncle in the mouse induced by cuprizone. J Neurol Sci [Internet]. 1973 [cited 2017 May 25];20(1):63-72. Available from: http://www.sciencedirect.com/science/ article/pii/0022510X73901184

16. Ludwin SK. Central nervous system demyelination and remyelination in the mouse: an ultrastructural study of cuprizone toxicity. Lab Invest [Internet]. 1978 Dec [cited 2017 May 25];39(6):597-612. Available from: http://www. ncbi.nlm.nih.gov/pubmed/739762.

17. Hiremath MM, Saito Y, Knapp GW, Ting JPY, Suzuki K, Matsushima GK Microglial/macrophage accumulation during cuprizone-induced demyelination in C57BL/6 mice. J Neuroimmunol. 1998;92:38-49.

18. Myhr K, Bø L. Model for Demyelination. Exp Neurol [Internet]. 2008; 117(4):3-4 Available from: https://doi.org/10.1016/.jexpneurol.2008.09.026.

19. Monteith S, Snell J, Eames M, Kassell NF, Kelly E, Gwinn R. Transcranial magnetic resonance-guided focused ultrasound for temporal lobe epilepsy: a laboratory feasibility study. J Neurosurg [Internet]. 2016 Dec [cited 2018 Jan 29];125(6):1557-64. Available from: http://www.ncbi.nlm. nih.gov/pubmed/26871207.

20. Jagannathan J, Sanghvi NT, Crum LA, Yen C-P, Medel R, Dumont AS, et al. High-intensity focused ultrasound surgery of the brain. Neurosurgery [Internet]. 2009 Feb [cited 2016 Dec 28];64(2):201-11. Available from: https:// www.ncbi.nlm.nih.gov/pubmed/?term=Jagannathan+J\%2C+Sanghvi + NT\%2C+Crum+LA\%2C

21. Jeong J-H, Hong GP, Kim Y-R, Ha J-E, Lee K-S. Clinical Consideration of Treatment to Ablate Uterine Fibroids with Magnetic Resonance Imaging-guided High Intensity Focused Ultrasound (MRgFUS): Sonalleve. J Menopausal Med [Internet]. 2016 Aug [cited 2018 Jan 29];22(2):94. Available from: http://www.ncbi.nIm.nih. gov/pubmed/27617244.
22. Marsac L, Chauvet D, Larrat B, Pernot M, Robert B, Fink M, et al. MR-guided adaptive focusing of therapeutic ultrasound beams in the human head. Med Phys [Internet]. 2012 Feb [cited 2018 Jul 28];39(2):1141-9. Available from: http://www.ncbi.nlm.nih.gov/pubmed/22320825.

23. Kim H, Chiu A, Lee SD, Fischer K, Yoo S-S. Focused ultrasound-mediated non-invasive brain stimulation: examination of sonication parameters. Brain Stimul [Internet]. 2014 Jul 2;7(5):748-56. Available from: http://www.ncbi. nlm.nih.gov/pmc/articles/PMC4167941/

24. Darvas F, Mehić E, Caler CJ, Ojemann JG, Mourad PD. Toward deep brain monitoring with superficial EEG sensors plus Neuromodulatory focused ultrasound. Ultrasound Med Biol. 2016:42(8):1834-47.

25. Barnett SB, Ter Haar GR, Ziskin MC, Rott H-D, Duck FA, Maeda K. International recommendations and guidelines for the safe use of diagnostic ultrasound in medicine. Ultrasound Med Biol [Internet]. 2000 [cited 2017 May 24];26(3):355-66. Available from: http://www.sciencedirect. com/science/article/pii/S0301562900002040

26. Yoo S-S, Kim H, Min B-K, Franck E, Park S. Transcranial focused ultrasound to the thalamus alters anesthesia time in rats. Neuroreport [Internet]. 2011 Oct 26 [cited 2018 Mar 19];22(15):783-7. Available from: https://insights.ovid. com/crossref?an $=00001756-201110260-00012$

27. Schindelin J, Arganda-Carreras I, Frise E. No Fiji: an open-source platform for biological image analysis. Nat Methods. 2012;9(7):676-82.

28. Baxter AG. The origin and application of experimental autoimmune encephalomyelitis. Nat Rev Immunol [Internet]. 2007 Nov; (november): 904-12. Available from: https://doi.org/10.1038/nri2190

29. Simmons SB, Pierson ER, Lee SY, Goverman JM. Modeling the heterogeneity of multiple sclerosis in animals. Trends Immunol [Internet]. 2013 Aug [cited 2018 Jul 28];34(8):410-22. Available from: http://linkinghub.elsevier.com/ retrieve/pii/S1471490613000690

30. Olechowski CJ, Tenorio G, Sauve Y, Kerr BJ. Changes in nociceptive sensitivity and object recognition in experimental autoimmune encephalomyelitis (EAE). Exp Neurol [Internet]. 2013;241(1):113-21 Available from: https://doi.org/10.1016/j.expneurol.2012.12.012.

\section{Ready to submit your research? Choose BMC and benefit from:}

- fast, convenient online submission

- thorough peer review by experienced researchers in your field

- rapid publication on acceptance

- support for research data, including large and complex data types

- gold Open Access which fosters wider collaboration and increased citations

- maximum visibility for your research: over $100 \mathrm{M}$ website views per year

At BMC, research is always in progress.

Learn more biomedcentral.com/submissions 